\title{
0588 ECONOMICS OF RISKY DRIVING: CROSS-SECTIONAL ANALYSIS OF EMERGENCY DEPARTMENT PATIENTS WITH HIGH-RISK BEHAVIOURS
}

B Sommers, J Fargo, J Shope, M Sommers* Correspondence: University of Pennsylvania, 231 Fagin Hall University of Pennsylvania Philadelphia, PA, 19104-4217, USA

\subsection{6/ip.2010.029215.588}

The concept of risky driving is not well articulated, nor is the societal burden imposed by risky driving behaviours. These behaviours lead to significant injury and mortality in the US and have application to global populations.

Aims We estimated the societal cost burden associated with risky driving, drinking-driving and substance use, among young adults exhibiting both high-risk driving behaviour and problem drinking.

Methods A sample of Emergency Department patients aged 18-44, who screened positive for risky driving and problem drinking ( $\mathrm{n}=313)$, were surveyed regarding driving behaviours, injuries, healthcare utilisation, legal problems and traffic crashes over the previous year. These self-reported data, supplemented by police crash reports, were used to estimate annual societal costs. Multivariate regressions were used to detect associations between costs and risky driving, drinkingdriving and substance use.

Results Societal costs related to driving behaviour and substance use averaged $\$ 23340$ per person, annually. Risky driving was consistently associated with higher criminal justice costs and total costs in univariate and multivariate models. Substance use was associated with increased injury, crash, and healthcare costs. Drinking-driving was not associated with increased costs in multivariate models. Compared to average drivers, the riskiest drivers imposed increased legal costs of $\$ 700$ and increased total societal costs of $\$ 2450$, annually. 
Conclusions Risky-driving imposes significant costs on society, even controlling for substance use and drinking-driving behaviour. Healthcare and other interventions to reduce riskydriving are likely to be both life-saving and cost-saving to society. 